\title{
Harmful effects of mechanical ventilation on neurocognitive functions
}

\author{
Federico Bilotta ${ }^{*}$ D, Giovanni Giordano, Paola Giuseppina Sergi and Francesco Pugliese
}

Whether mechanical ventilation (MV) induces neurotoxicity that can trigger or accelerate chronic cognitive disorders is controversial $[1,2]$. The relationship between MV and neurocognitive impairment-that persisted at hospital discharge and at 1-year follow up-was first reported in 1999 in MV-treated ARDS patients [3]. Since then, several preclinical and clinical studies have investigated the mechanisms, localization, and timing of brain damage induced by MV and possible preventive/therapeutic strategies.

\section{MV-induced brain damage: mechanisms, localization, and timing}

Alveolar stretching, $\mathrm{PaO}_{2} / \mathrm{PaCO}_{2}$ abnormalities, and cytokine and chemokine expression in the lungs are the pivotal mechanisms that possibly contribute to MV-induced brain damage [4-9] (Table 1). Alveolar stretching promotes the production of systemic and cerebral mediators (cytokines, chemokines, reactive oxygen species, microglia, and immune system activation) that lead to neuroinflammation, dopaminergic neuronal signaling, and beta amyloid synthesis [4-9]. Relevance of MV-induced alveolar stretching in brain damage is witnessed by production of cerebral betaamyloid and Alzheimer's disease-like brain degeneration (cerebral amyloid $\beta$ peptide accumulation, neuroinflammation, and blood-brain barrier dysfunction) even after relatively "short-term MV" (after $4 \mathrm{~h}$ MV in mice) - as reported in a recent study published in Critical Care [8]. The $\mathrm{PaO}_{2} / \mathrm{PaCO}_{2}$ abnormalities-including hypo and hyper $\mathrm{O}_{2}$ and/or $\mathrm{CO}_{2}$ that often represent the indication to establish $\mathrm{MV}$ and can persist during $\mathrm{MV}-$ might contribute to promote neuronal death, cerebral oxidative stress, and changes in brain blood flow that worsen brain damage $[10,11]$. Increased systemic inflammatory mediators produced by the lungs during MV ("biotrauma") have been detected in several preclinical studies and include TNF alpha, IL6, IL10, IL1 beta, MCP1, and MIP2, which have a proven

\footnotetext{
* Correspondence: bilotta@tiscali.it

Department of Anesthesiology, Critical Care and Pain Medicine, Sapienza University of Rome, Rome, Italy
}

neuroinflammatory role $[4,5,7]$. Furthermore, MV directly triggers brain inflammation inducing cerebral expression of proinflammatory cytokines (TNF-alpha, IL-1beta, and IL-6) and microglia activation $[6,7,11]$. Of note, the increase in some cerebral inflammatory mediators (TNFalpha) is proven to be higher when larger tidal volume is applied [5]. Also, the immune system modulation has a key role in MV-induced neuroinflammation, and in preclinical models, an increase in Toll-like receptor 4 (TLR-4) expression (a component of the innate immune system with a role in neuronal survival, neuroplasticity, memory, learning, and cognitive decline) has been detected in the hippocampus after $6 \mathrm{~h}$ of MV [7]. Of note, the increase in brain TLR4 expression, and the related increase in plasma and hippocampus cytokines (IL1beta, IL6, TNF alpha) concentration, predicts the extent of memory impairment [7]. The lung-brain vagal reflex induced by MV can also contribute activating dopamine receptors type 2 (DRD2) in the hippocampus and leading to neuronal apoptosis [9].

Some evidence brought conflicting results on MV-induced neurocognitive decline. While alveolar stretch induced by MV promotes de novo synthesis of adhesion molecules in the lung, liver, and kidneys, there is no evidence of such effect in the brain [12]. Furthermore, several of the studies that tested the impact of MV on the brain and neurocognitive functions have limitations that make recorded results not immediately suitable for the clinical realm and lack to discriminate for possibly confounding factors. Last but not the least, the histo-pathological damage, especially when it occurs in the brain, does not necessarily translate into a clinically detectable impairment of neurocognitive functions. For example, a study from Kamuf and coll showed no relationship between MV and an increase in inflammatory mediators eventually associated with brain injury [6].

Clinical relevance of $\mathrm{PaO}_{2} / \mathrm{PaCO}_{2}$ abnormalities and their impact on cognitive function in MV-treated patients has been reported by several studies [10, 13]. Hypoxiafrequent in patients in whom MV is indicated-has been hypothesized to be the most relevant cause of long-term cognitive impairment in patients with ARDS, and it is 
Table 1 Mechanisms of MV-induced brain damage

\begin{tabular}{lll}
\hline $\begin{array}{l}\text { Alveolar } \\
\text { stretching }\end{array}$ & $\begin{array}{l}\text { Systemic and cerebral } \\
\text { mediators of } \\
\text { neuroinflammation }\end{array}$ & $\begin{array}{l}\text { Systemic release of lung-produced } \\
\text { inflammatory mediators ("biotrauma") } \\
\text { Local activation of brain } \\
\text { inflammatory mediators } \\
\text { Immunomediated: Toll-like } \\
\text { receptor expression }\end{array}$ \\
& $\begin{array}{l}\text { Afferent neuronal signal: DRD2 } \\
\text { Beta amyloid production }\end{array}$ \\
$\mathrm{PaO}_{2} / \mathrm{PaCO}_{2}$ & Hypo/hyperoxia \\
abnormalities & \\
& Hypo/hypercapnia & \\
\hline
\end{tabular}

Alveolar stretching-through mechanotransduction-induces neuroinflammation with the release of mediators

associated with increased mortality in patients with acute brain damage that require $\operatorname{MV}[3,13]$. It affects neuronal oxidative phosphorylation, and some brain areas-as the hippocampus-result to be more vulnerable to hypoxic damage and are keener to develop atrophy after hypoxic events [13]. Hypercapnia-another possible indication for MV-causes cerebral vasodilatation with consequent increase of intracranial pressure, and it is associated with mild cognitive impairment in MV preclinical and clinical models $[3,13]$. On the other hand, also hyperoxia-which sometime occurs during $\mathrm{MV}$-is a possible mechanism of brain damage since it impairs cerebral autoregulation, reduces $\mathrm{CMRO}_{2}$, and is associated with delayed visual evoked potentials $[3,13]$. Hypocapnia-a possible iatrogenic complication of $\mathrm{MV}$-induces cerebral vasoconstriction, reduces cerebral compliance, and is associated with a poorer neurological outcome in MV experimental models $[10,13]$.

\section{Monitoring and prevention of MV-induced neurocognitive functions decline}

Several strategies have been tested to prevent MV-induced brain damage and the related neurocognitive decline; these include neuroinflammatory scavengers, immune modulation, to blunt the dopaminergic neuronal signaling with DRD2-blockers, and lung protective ventilation strategies. The use of scavenger receptor class A has been extensively tested in Alzheimer disease and might play a role in the prevention of $\mathrm{MV}$-induced neurocognitive functions decline. Immune modulation with TLR-4 antagonist has been proven in preclinical models to effectively reduce MV-related neuroinflammation and the extent of cognitive disorders [7]. The use of selective DRD2-blocker (haloperidol) has been successfully tested in experimental animals and is proven to restore the activity of cell survival pathways in the hippocampus, potentially reducing neuronal apoptosis [9]. Protective lung ventilation, with the use of low tidal volume in patients hospitalized after resuscitation for cardiac arrest, associates with better neurocognitive outcome [14]. Optimal $\mathrm{PaO}_{2} / \mathrm{PaCO}_{2}$ management correlates with better neurocognitive status in patients with acute brain injury treated with MV $[10,13]$.

\section{Conclusions}

Growing preclinical and clinical evidence suggest that MV induces harmful effects on neurocognitive functions. It is not easy to discriminate whether these changes should be interpreted as direct MV-induced damage or as result of perioperative/critical care environment and preexisting frailty. Given the importance to preserve patient's cognitive functions, we consider it necessary to define a workup dedicated to monitor neuroinflammation during MV that might include neuroinflammatory scavengers, immune modulation, DRD2-blockers, and lung "neuroprotective" MV management. The latter should encompass optimal MV setting, including low tidal volume, the lowest $\mathrm{FiO}_{2}$ needed, to maintain-when possible- $-\mathrm{PaCO}_{2}$ within normal values and to warrant the shortest MV exposure.

\begin{abstract}
Abbreviations
ARDS: Acute respiratory distress syndrome; DRD2: Dopamine receptor D2; $\mathrm{FiO}_{2}$ : Fraction of inspired oxygen; ICU: Intensive care unit; IL-10: Interleukin 1; IL-1 beta: Interleukin 1-beta; IL-6: Interleukin 6; MCP-1: Monocyte chemoattractant protein; MIP-2: Macrophage inflammatory protein; MV: Mechanical ventilation; $\mathrm{PaCO}_{2}$ : Arterial partial pressure of carbon dioxide; $\mathrm{PaO}_{2}$ : Arterial partial pressure of oxygen; TLR4: Toll-like receptor 4; TNFalpha: Tumor necrosis factor-alpha
\end{abstract}

\section{Authors' contributions}

All authors participated in drafting the manuscript. All authors read and approved the final manuscript.

Funding

None.

Availability of data and materials

Not applicable.

Ethics approval and consent to participate

Not applicable.

Consent for publication

All authors have reviewed the final version of the manuscript and approve the manuscript for publication.

\section{Competing interests}

The authors declare that they have no competing interests.

Received: 22 March 2019 Accepted: 18 July 2019

Published online: 06 August 2019

\section{References}

1. Pelosi P, Rocco PR. The lung and the brain: a dangerous cross-talk. Crit Care. 2011:15(3):168.

2. Kamuf J, Garcia-Bardon A, Ziebart A, Thomas R, Folkert K, Frauenknecht K, Thal SC, Hartmann EK. Lung injury does not aggravate mechanical ventilation-induced early cerebral inflammation or apoptosis in an animal model. PLoS One. 2018 9;13(8):e0202131.

3. Hopkins RO, Weaver LK, Pope D, Orme JF, Bigler ED, Larson LV. Neuropsychological sequelae and impaired health status in survivors of severe acute respiratory distress syndrome. Am J Respir Crit Care Med. 1999;160:50-6. 
4. Bickenbach J, Zoremba N, Fries M, et al. Low tidal volume ventilation in a porcine model of acute lung injury improves cerebral tissue oxygenation. Anesth Analg. 2009;109(3):847-55.

5. Quilez ME, Fuster G, Villar J, et al. Injurious mechanical ventilation affects neuronal activation in ventilated rats. Crit Care. 2011;15:R124.

6. Kamuf J, Garcia-Bardon A, Ziebart A, et al. Lung injury does not aggravate mechanical ventilation-induced early cerebral inflammation or apoptosis in an animal model. PLoS One. 2018;13(8):e0202131.

7. Chen T, Chen C, Zhang Z, Zou Y, Peng M, Wang Y. Toll-like receptor 4 knockout ameliorates neuroinflammation due to lung-brain interaction in mechanically ventilated mice. Brain Behav Immun. 2016;56:42-55.

8. Lahiri S, Regis GC, Koronyo Y, et al. Acute neuropathological consequences of short-term mechanical ventilation in wild-type and Alzheimer's disease mice. Crit Care. 2019;23(1):63.

9. González-López A, López-Alonso I, et al. Mechanical ventilation triggers hippocampal apoptosis by vagal and dopaminergic pathways. Am J Respir Crit Care. 2013; Med;188:693-702

10. Badenes R, Bilotta F. Neurocritical care for intracranial haemorrhage: a systematic review of recent studies. Br J Anaesth. 2015;115(Suppl 2):ii68-74.

11. Chen C, Zhang Z, Chen T, Peng M, Xu X, Wang Y. Prolonged mechanical ventilation-induced neuroinflammation affects postoperative memory dysfunction in surgical mice. Crit Care. 2015;10(19):159.

12. Hegeman MA, Hennus MP, Heijnen CJ, Specht P, Lachmann B, Jansen N, Van Vught J, Cobelens PM. Ventilator-induced endothelial activation and inflammation in the lung and distal organs. Crit Care. 2009;13(6):R182.

13. Borsellino B, Schultz MJ, Gama de Abreu M, Robba C, Bilotta F. Mechanical ventilation in neurocritical care patients: a systematic literature review. Expert Rev Respir Med. 2016;10(10):1123-32.

14. Beitler JR, Ghafouri TB, Jinadasa SP, et al. Favorable neurocognitive outcome with low tidal volume ventilation after cardiac arrest. Am J Respir Crit Care Med. 2017;195(9):1198-206.

\section{Publisher's Note}

Springer Nature remains neutral with regard to jurisdictional claims in published maps and institutional affiliations. 